\title{
INFORMAÇÕES EDITORIAIS 2010
}

\begin{tabular}{lcccccc}
\hline \multicolumn{7}{c}{ ARTIGOS PUBLICADOS } \\
\hline Edições & Número de Artigos & Artigos por & Artigos \\
& EfPR & $\%$ & OUTRAS & $\%$ & Reprão & Reprovados \\
Jan/Abr, 2010 & & 0 & 5 & 100 & 5 & 3 \\
\hline Mai/Ago, 2010 & 1 & 16,67 & 5 & 83,33 & 6 & 4 \\
Set/Dez, 2010 & 1 & 16,67 & 5 & 83,33 & 6 & 6 \\
Média & 0,67 & 11,11 & 5 & 88,24 & 5,67 & 4,33 \\
\hline
\end{tabular}

\section{AUTORIA DOS ARTIGOS}

\begin{tabular}{lcccc}
\hline Edições & \multicolumn{3}{c}{ Número de Autores } & Total de Autores \\
& UFPR & PR & OUTRAS UF's & \\
Jan/Abr, 2010 & & & 11 & 11 \\
\hline Mai/Ago, 2010 & 3 & 3 & 14 & 17 \\
Set/Dez, 2010 & 1 & 8 & 12 & 20 \\
\hline Média/total & $\mathbf{1 , 3 3 3 3}$ & $\mathbf{3 , 6 7}$ & $\mathbf{1 2 , 3 3}$ & $\mathbf{4 8}$ \\
\hline
\end{tabular}

\section{DEMANDA POR PUBLICAÇÃO}

\begin{tabular}{lcc}
\hline Artigos & \multicolumn{2}{c}{ Período Jan. a Dez./2010 } \\
Recebidos & 36 & 100 \\
Aprovados & 17 & $47,22 \%$ \\
Não Aprovados (após Blind review) & 10 & $27,78 \%$ \\
Não aceitos para avaliação & 3 & $8,33 \%$ \\
Em avaliação & 6 & $16,67 \%$ \\
\hline
\end{tabular}




\section{$\mathrm{R} C \& \mathrm{C}$}

REVISTA DE CONTABILIDADE E CONTROLADORIA

\begin{tabular}{lcccccc}
\hline \multicolumn{3}{c}{ NUMERO DE AUTORES POR UNIDADE DE FEDERAÇÃO } \\
\hline \multicolumn{1}{c}{ Edições } & $\begin{array}{c}\text { Jan/Abr, } \\
\text { 2010 }\end{array}$ & \multicolumn{3}{c}{$\begin{array}{c}\text { Mai/Ago, } \\
\text { 2010 }\end{array}$} & \multicolumn{3}{c}{ Set/Dez, } \\
& $F i$ & $F i \%$ & $F i$ & $F i \%$ & $F i$ & $F i \%$ \\
Paraná & & 0,00 & 3 & 17,65 & 8 & 40,00 \\
Santa Catarina & & 0,00 & & 0,00 & 5 & 25,00 \\
Porto Alegre & 3 & 27,27 & 4 & 23,53 & & 0,00 \\
Minas Gerais & 2 & 18,18 & 4 & 23,53 & 3 & 15,00 \\
Pernambuco & & 0,00 & 4 & 23,53 & & 0,00 \\
Rio de Janeiro & 2 & 18,18 & & 0,00 & & 0,00 \\
São Paulo & 4 & 36,36 & & 0,00 & 3 & 15,00 \\
Distrito Federal & & 0,00 & 2 & 11,76 & 1 & 5,00 \\
Total & $\mathbf{1 1}$ & 100,00 & $\mathbf{1 7}$ & 100,00 & $\mathbf{2 0}$ & 100,00 \\
\hline
\end{tabular}

\begin{tabular}{|c|c|c|c|c|}
\hline \multicolumn{4}{|c|}{ INSTITUIÇÕES DE ORIGEM DOS ARTIGOS } & \\
\hline \multirow[t]{2}{*}{ UF de origem } & \multicolumn{4}{|c|}{ Edições } \\
\hline & Jan/Abr, 2010 & Mai/Ago, 2010 & Set/Dez, 2010 & TOTAL \\
\hline UFPR-PR & & 1 & 1 & 2 \\
\hline PUC-PR & & & 1 & 1 \\
\hline UPS-SP & & & 1 & 1 \\
\hline FURB-SC & & & 1 & 1 \\
\hline UFMG-MG & 1 & 1 & 1 & 3 \\
\hline UNISSUL-SC & & & 1 & 1 \\
\hline UFPE-PE & & 1 & & 1 \\
\hline UNB-DF & & 1 & & 1 \\
\hline UNISINOS-RS & 1 & 1 & & 2 \\
\hline UFL-MG & & 1 & & 1 \\
\hline UERJ-RJ & 1 & & & 1 \\
\hline SANTO ANDRE-SP & 1 & & & 1 \\
\hline MACKENZIE-SP & 1 & & & 1 \\
\hline
\end{tabular}




AVALIADORES BRASILEIROS AD HOC E INSTITUIÇÃO DE
VÍNCULO

\begin{tabular}{|c|c|}
\hline Avaliadores Brasileiros & Instituição \\
\hline Ana Paula Mussi Szabo Cherobim & Universidade Federal do Paraná \\
\hline Aneide de Oliveira Araujo & Universidade Federal do Rio Grande do Norte \\
\hline Antônio Artur de Souza & Universidade Federal de Minas Gerais \\
\hline Antonio Carlos Dias Coelho & Universidade Federal do Ceará \\
\hline Antonio Henriques de Araújo & Instituto Tecnológica da Aeronáutica \\
\hline Antonio Lopo Martinez & Fucape Business School \\
\hline Aracéli Cristina de Sousa Ferreira & Universidade Federal do Rio de Janeiro \\
\hline Carlos Renato Theóphilo & Universidade Estadual de Montes Claros \\
\hline Cláudio Parisi & Fundação Escola de Comércio Álvares Penteado \\
\hline Edgard Bruno Cornachione Junior & Universidade de São Paulo \\
\hline Eliseu Martins & Universidade de São Paulo \\
\hline Ernani Ott & Universidade do Vale do Rio dos Sinos \\
\hline Fábio Frezatti & Universidade de São Paulo \\
\hline Gilmar Almeida Gomes & Universidade do Estado de Santa Catarina \\
\hline Ilse Maria Beuren & Universidade Regional de Blumenau \\
\hline Jacqueline Veneroso Alves da Cunha & Universidade Federal de Minas Gerais \\
\hline José Alonso Borba & Universidade Federal de Santa Catarina \\
\hline Jose Luis Gomes da Silva & Universidade de Taubaté \\
\hline José Maria Dias Filho & Universidade Federal da Bahia \\
\hline Lauro Brito de Almeida & Universidade Federal do Paraná \\
\hline Léo Tadeu Robles & Pontificia Universidade Católica de Santos \\
\hline Luiz Carlos Miranda & Universidade Federal de Pernambuco \\
\hline Maísa de Souza Ribeiro & Universidade de São Paulo \\
\hline Márcia Martins Mendes De Luca & Universidade Federal do Ceará \\
\hline Marcos Antonio de Souza & Universidade do Vale do Rio dos Sinos \\
\hline Mariomar de Sales Lima & Universidade Federal do Amazonas \\
\hline Masayuki Nakagawa & Universidade de São Paulo \\
\hline Moacir Sancovschi & Universidade Federal do Rio de Janeiro \\
\hline Monica Franchi Carniello & Universidade de Taubaté \\
\hline Olga Maria Panhoca da Silva & Universidade do Estado de Santa Catarina \\
\hline Paulo Arnaldo Olak & Universidade Estadual de Londrina \\
\hline Paulo Roberto Barbosa Lustosa & Universidade Federal de Brasília \\
\hline
\end{tabular}


AVALIADORES ESTRANGEIROS AD HOC

\begin{tabular}{|l|l|l|l|}
\hline Avaliadores estrangeiros & Instituição & Cidade & País \\
\hline Carlos Alberto de Mello-e-Souza & Seattle University & Washington & Estados Unidos \\
\hline Francisco Carreira & Instituto Politécnico de Setúbal & Setúbal & Portugal \\
\hline
\end{tabular}

INSTITUIÇÕES DE LOTAÇÃO DOS AVALIADORES BRASILEIROS

\begin{tabular}{|l|c|c|c|}
\hline \multicolumn{1}{|c|}{ Instituição } & Estado & Quantidade & Percentual \\
\hline Fucape Business School & ES & 1 & 3,03 \\
\hline Fundação Escola de Comércio Álvares Penteado & SP & 1 & 3,03 \\
\hline Instituto Tecnológica da Aeronáutica & SP & 1 & 3,03 \\
\hline Pontificia Universidade Católica de Santos & SP & 1 & 3,03 \\
\hline Universidade de São Paulo & SP & 6 & 18,18 \\
\hline Universidade de Taubaté & SP & 2 & 6,06 \\
\hline Universidade do Estado de Santa Catarina & SC & 2 & 6,06 \\
\hline Universidade do Vale do Rio dos Sinos & RS & 2 & 6,06 \\
\hline Universidade Estadual de Londrina & PR & 1 & 3,03 \\
\hline Universidade Estadual de Montes Claros & MG & 1 & 3,03 \\
\hline Universidade Federal da Bahia & BA & 1 & 3,03 \\
\hline Universidade Federal de Brasília & DF & 1 & 3,03 \\
\hline Universidade Federal de Minas Gerais & MG & 2 & 6,06 \\
\hline Universidade Federal de Pernambuco & PE & 1 & 3,03 \\
\hline Universidade Federal de Santa Catarina & SC & 1 & 3,03 \\
\hline Universidade Federal do Amazonas & AM & 1 & 3,03 \\
\hline Universidade Federal do Ceará & CE & 2 & 6,06 \\
\hline Universidade Federal do Paraná & PR & 2 & 6,06 \\
\hline Universidade Federal do Rio de Janeiro & RJ & 2 & 6,06 \\
\hline Universidade Federal do Rio Grande do Norte & RN & 1 & 3,03 \\
\hline Universidade Regional de Blumenau & SC & 1 & 3,03 \\
\hline
\end{tabular}


R C \& C

REVISTA DE CONTABILIDADE E CONTROLADORIA

LOCAL DE ORIGEM DOS AVALIADORES

\begin{tabular}{|c|c|c|}
\hline Estado & Quantidade & Percentual \\
\hline AM & 1 & 3,03 \\
\hline BA & 1 & 3,03 \\
\hline CE & 2 & 6,06 \\
\hline DF & 1 & 3,03 \\
\hline ES & 1 & 3,03 \\
\hline MG & 3 & 9,09 \\
\hline PE & 1 & 3,03 \\
\hline PR & 3 & 9,09 \\
\hline RJ & 2 & 6,06 \\
\hline RN & 1 & 3,03 \\
\hline RS & 2 & 6,06 \\
\hline SC & 4 & 12,12 \\
\hline SP & 11 & 33,33 \\
\hline
\end{tabular}

Total

33

100,00 